\title{
Measurement of Time Dependent Fields in High Gradient Superconducting Quadrupoles for the Tevatron *
}

\author{
M. J. Lamm, K. Coulter, S. Gourlay and T. S. Jaffery \\ Fermi National Accelerator Laboratory \\ P.O. Box 500 \\ Batavia, Illinois 60510
}

October 1990

* Presented at the 1990 Applied Superconductivity Conference, Snowmass, Colorado, September 24-28, 1990. 
MEASUREMENT OF TIME DEPENDENT FIELDS IN HIGH GRADIENT SUPERCONDUCTING QUADRUPOLES FOR THE TEVATRON

\author{
M.J. Lamm, K. Coulter, S. Gourlay, T. S. Jaffery \\ Fermi National Accelerator Laboratory \\ P.O. Box 500 \\ Batavia, Illinois 60510
}

\section{Abstract}

Magnetic field measurements have been performed on prototype and production magnets from two high gradient superconducting quadrupoles designs. One design is a double shell quadrupole with 36 strand Rutherford cable. The other design is a single shell quadrupole with 5 individually insulated monolithic strands connected in series. These magnets have similar bore diameters and cable dimensions. However, there are significant differences between the two designs, as well as differences between prototype and production magnets within each design, with regard to $\mathrm{Cu}$ to superconductor ratio, filament diameter and filament spacing to strand diameter. The time dependence at fixed currents of the measured magnetic fields is discussed.

\section{Introduction}

Two high gradient superconducting quadrupoles designs were built and tested for the Tevatron Low Beta DO/BO insertion 1 . Measurements of time dependent magnetic fields at fixed excitation currents were included in the evaluation of some of the production magnets as well as one of the prototype magnets. These tests were patterned after previously reported 2 studies performed on Tevatron dipole magnets. In these studies it was observed that magnetic field decays were a mixture of linear and non-linear components in $\log (t)$ and this dependence was a function of the current excitation history. The $\log (t)$ nature of the decay is indicative of "flux creep", but contributions from other processes could not be ruled out. In this paper we present evidence for $\log (t)$ and exponential time decays in these quadrupole magnets.

\section{Description of magnets.}

A complete description of each of the two magnet designs has been reported elsewhere 3,4,5. Both magnet designs have cold iron, aluminum collars and a $7.54 \mathrm{~cm}$ aperture. One design is a 2-shell $\cos$ (2-theta) quadrupole with 36 strand NbTi Rutherford style cable. The strand consists of 612 filaments of 13 micron diameter and has a $\mathrm{Cu} /$ superconductor ratio of $1.5 / 1$. These 2-shell quadrupoles were built in four magnetic lengths to satisfy the Low Beta Insertion lattice requirements. The field gradient is $1.41 \mathrm{~T} / \mathrm{cm}$ at the maximum operating current of $4832 \mathrm{~A}$. The other design is a 1 -shell $\cos (2-$ theta) quadrupole with a $61 \mathrm{~cm}$ magnetic length. The coil has a geometry similar to the 2-shell magnet inner coil but the cable consists of five monolithic strands individually wrapped in kapton. The strands are connected in series thus producing a high gradient magnet with modest current requirements $(.582 \mathrm{~T} / \mathrm{cm}$ at the maximum operating current of $1000 \mathrm{amps}$ ). A 1-shell prototype was built with the same coil geometry as the 1-shell production magnets but with significantly different strands parameters. For example the production strands consists of $612 \mathrm{NbTi}$ filaments of 44 micron diameter while the prototype has $2120 \mathrm{NbTi}$ filaments with a diameter of 20 microns. The prototype also has a much larger $\mathrm{Cu} /$ superconductor ratio (1.8 vs 1.5$)$ and consequently a larger filament spacing/diameter.

\section{Definition of Field Harmonics}

The magnetic field can be described by the following definition:

$$
B_{x}-i B_{y}=\sum_{n=0}\left(A_{n}-i B_{n}\right) *\left((x+i y) / R_{0}\right)^{n}
$$

Manuscript received September 24, 1990.
$A_{n}$ and $B_{n}$ are the skew and normal coefficients and $R_{0}$ is the reference radius chosen to be $2.54 \mathrm{~cm}$ (1 inch) for the Tevatron. These coefficients have units of Gauss evaluated at the reference radius $R_{0}$. For the purpose of these tests the $x$ and $y$ directions are chosen so that the skew quadrupole term is zero for non-zero transport currents. This introduces a two-fold ambiguity due to the two-fold current symmetry. For a perfectly constructed quadrupole, the skew terms vanish and only normal terms with $n=4 M+1$ $(M=0,1,2 .$.$) can exist. Thus the first allowed harmonic for a$ quadrupole is the duodecapole $(n=5)$. For fields due to non-zero transport currents, it is customary to express the magnetic field coefficients as $10^{-4}$ times the ratio of the multipole field at the reference radius, $2.54 \mathrm{~cm}$, to the quadrupole field gradient. These "normalized" coefficients are denoted by the symbols $a_{n}, b_{n}$ for skew and normal coefficients respectively.

\section{Experimental Details}

\section{Test Facilities}

Magnets were tested in the $2.4 \mathrm{~m}$ vertical liquid helium dewar located in the Superconducting Magnet R\&D facility at Fermilab. The dewar is instrumented with pressure transducers and liquid helium level gages. The shell of each magnet was instrumented with Carbon thermometers which were calibrated to better than $10 \mathrm{mK}$. Current was supplied by a Transrex 500-5 power supply. The current was monitored through a $10 \mu \mathrm{ohm}$ precision shunt. Cryogenic and quench data were collected with a CAMAC data acquisition system patterned after the system used in the Fermilab Magnet Test facility 7,8 Magnetic measurements were performed with $46 \mathrm{~cm}$ long multiple winding Morgan coil inserted into an anti-cryostat which, in turn, was inserted into the bore of the magnet. The anti-cryostat consists of two concentric seemless stainless steel shells. The space in between the two shells was filled with superinsulation. The Morgan coil was rotated at approximately $6 \mathrm{~Hz}$. and the resultant voltages were processed by a V/F based magnetic measuring system 9 .

\section{Tests Description}

Magnets were tested using two types of current excitation cycles. In all cases, the magnet was quenched prior to the test cycle to remove any remnant fields due to persistent currents.

In the first cycle, magnet was ramped in a sawtooth pattern several times at a fixed ramp rate. On the last up-ramp, the ramp was stopped at 300 amps and was held at this current for $500-1600 \mathrm{sec}$. The final ramp was then completed at the previous ramp rate. Harmonics data were recorded during the last two ramps including the period of constant current. This cycle simulates an injection front porch like the one used for Tevatron collider operation.

The second cycle was designed to study the remnant magnetic fields. The magnets are first ramped in a sawtooth pattern several times at a fixed ramp rate. Hamonics data were recorded on the final ramp as well as $900-1800$ seconds after the magnet reaches zero current.

Because of the possible sensitivity of time dependent magnetic fields to fluctuations in temperature and transport current, care was taken to monitor these parameters during each magnet tests. Helium bath temperature, as well as dewar pressure were recorded in 60-120 second intervals before, during and after the ramp cycle. The magnet temperature varied by less than $10 \mathrm{mK}$ over a typical 1000 second run. Transport current was measured in coincidence with every magnetic measurement. During a typical $25 \mathrm{~A} / \mathrm{s}$ ramp to a $300 \mathrm{amp}$ constant current, there was no detectable current overshoot. The transformation from up-ramp to constant current occurred in 1-2 
seconds and had the shape of a parabolic rolloff. The average point to point scatter at constant current was less than $100 \mathrm{~mA}$. Long term current drift over 500 seconds was less than $200 \mathrm{~mA}$. During remnant field studies no detectable current overshoot, point to point scatter or current drift was observed.

\section{Harmonic Measurements}

\section{2-Shell Quadrupole}

A $60 \mathrm{~cm}$ 2-shell quadrupole was tested using the $300 \mathrm{amp}$ "injection" cycle. The ramp rate was $50 \mathrm{~A} / \mathrm{s}$ and the maximum sawtooth current was 2000 amps. Six ramps were executed prior to the final ramp containing a 1800 second period of constant current. Figure 1 shows bs vs current in the region of 300 amps for the sixth ramp and the final ramp with the period of constant current. As the current approached 300 amps, b5 became more positive with respect to the previous bs vs current hysteresis curve. This bs drift continued while the current remained constant. When the up-ramp was continued, bs rejoined the previous b5 curve after approximately 100 amps ( 2 seconds). The time dependence of bs during the period of constant current is shown in figure 2 where $t=0$. We observed a monotonic increase in $b_{5}$ that approximates $\log (t)$ behavior.

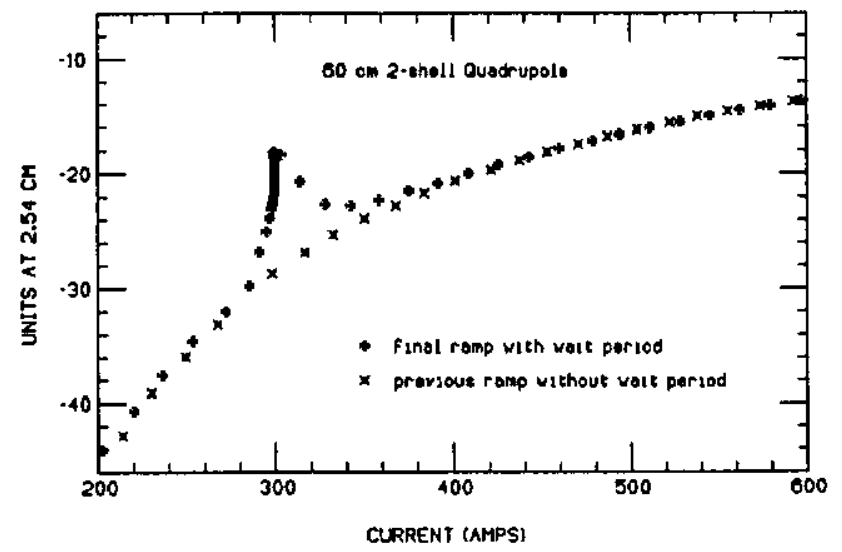

Figure 1. Normalized 12 pole (b5) as a function of current near 300 amps for 2-shell quadrupoles during an "injector" style ramp cycle. The final ramp cycle during the $1800 \mathrm{sec}$ wait period exhibits time dependent $b 5$ changes compared to the previous uninterrupted cycle.

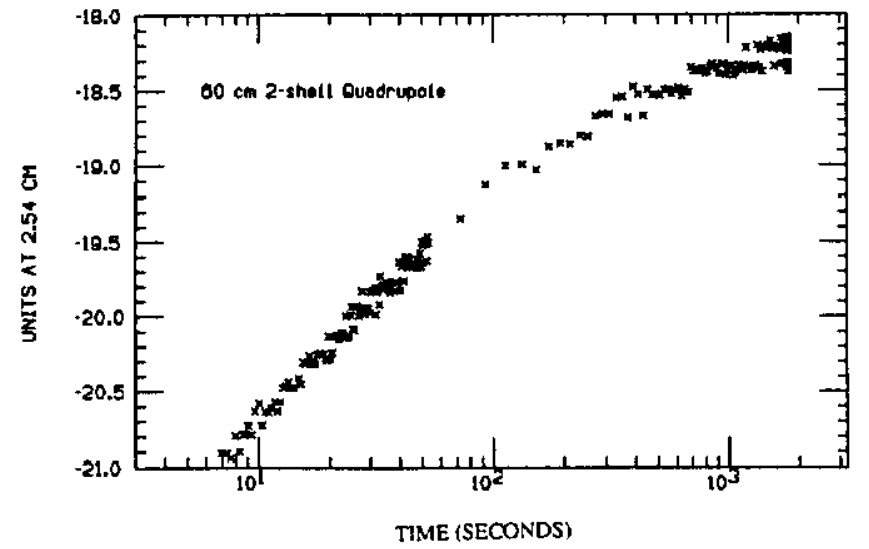

Figure 2. Normalized 12 pole $\left(b_{5}\right)$ as a function of time. $t=0$ is defined as the beginning of the $1800 \mathrm{sec}$ period of $300 \mathrm{amp}$ current.

Tests were also performed using the remnant field cycle. The maximum sawtooth current was 4800 amps. We recorded the harmonics data during the sixth (final) sawtooth ramp and during the first 1800 seconds after the magnet reached zero current. Figure 3 shows $B_{5}$ and $B 9$ as a function of $\log (t)$ where $t=0$ is defined as the beginning of zero current. Again the decay of $\mathrm{B}_{5}$ and $\mathrm{B}_{9}$ approximates $\log (t)$ behavior. However, in both tests the magnetic field time dependence is inconsistent with a simple $\log (t)$ dependence.

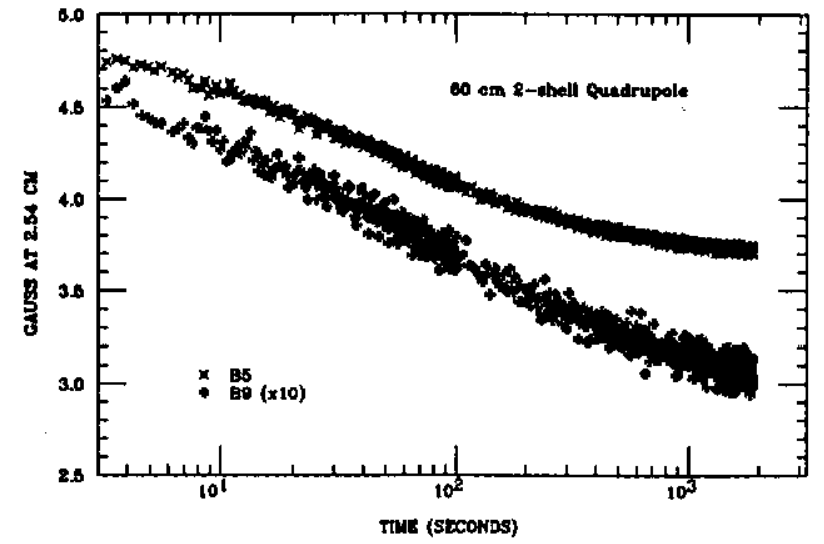

Figure 3. Remnant 12 pole $\left(B_{5}\right)$ and 20 pole $\left(B_{9}\right)$ as a function of time. $t=0$. is defined as the time when the transport current reaches zero current.

\section{1-Shell Magnets}

Both injection cycle and remnant field cycles were recorded on one prototype and one production magnet. For the injection cycle tests, the magnets are ramped four times to a maximum current of 1500 amps, prior to the final ramp containing a 500 second period of constant current at 300 amps. Figure 4 shows b5 vs current near 300 amps for the production and the prototype magnet. The ramp rate for this cycle was $25 \mathrm{~A} / \mathrm{s}$. The shape of the observed deviations of b5 from the nominal bs hysteresis curve are very different than those observed on the 2-shell magnet.
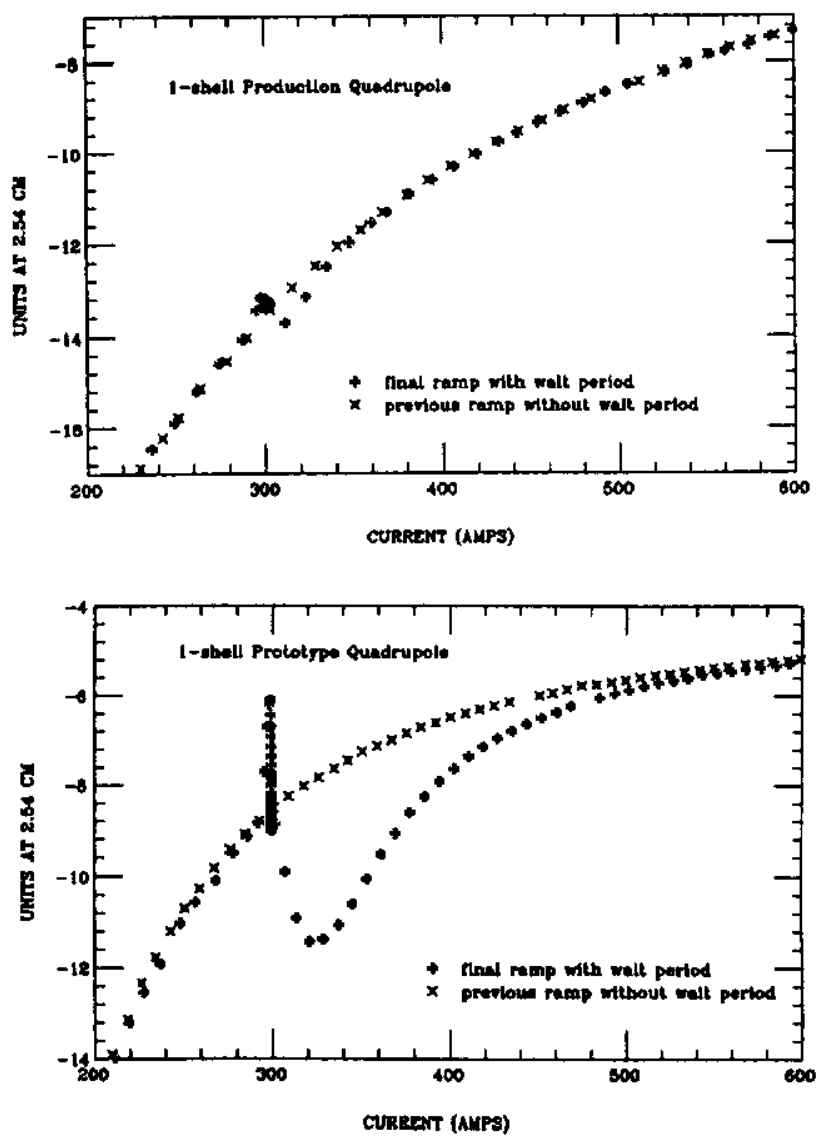

Figure 4. Normalized 12 pole (b5) as a function of current near 300 amps for a) production b) prototype 1-shell quadrupoles during $25 \mathrm{~A} / \mathrm{s}$ "injector" style ramp cycle. The final ramp cycle with the 1800 sec. wait period exhibits time dependent bs changes compared to the previous uninterrupted cycle. 
The time dependence of this 12-pole deviation for the prototype magnet is shown in figure 5 . The $\mathrm{B}_{5}$ contribution from the last uninterrupted ramp cycle is subtracted from the measured $B_{5}$ from the cycle with the 500 second period of constant current, and the resultant distribution is plotted as a function of time. Figure 5a shows the $\mathrm{B}_{5}$ difference at the beginning of the "wait period" and Figure $5 \mathrm{~b}$ shows $B_{5}$ at the beginning of the up-ramp for the prototype magnets. $\mathrm{B} \dot{\xi}$ increased in approximately 1 second followed by a decay with a time constant of approximately 3 seconds. Then $B_{5}$ increased logarithmically while the current remained constant. When the upramp was started, $B_{5}$ decreased suddenly to a value below the uniterrupted hysteresis curve, and then rejoins the hysteresis curve with a time constant of approximately 3 seconds. The magnitude of this second exponential decay is approximately twice of that observed at the begining of the wait period. In other studies, the magnitude of both exponential decays increased with increasing ramp rate. A similar but much smaller effect was observed on the production magnet.
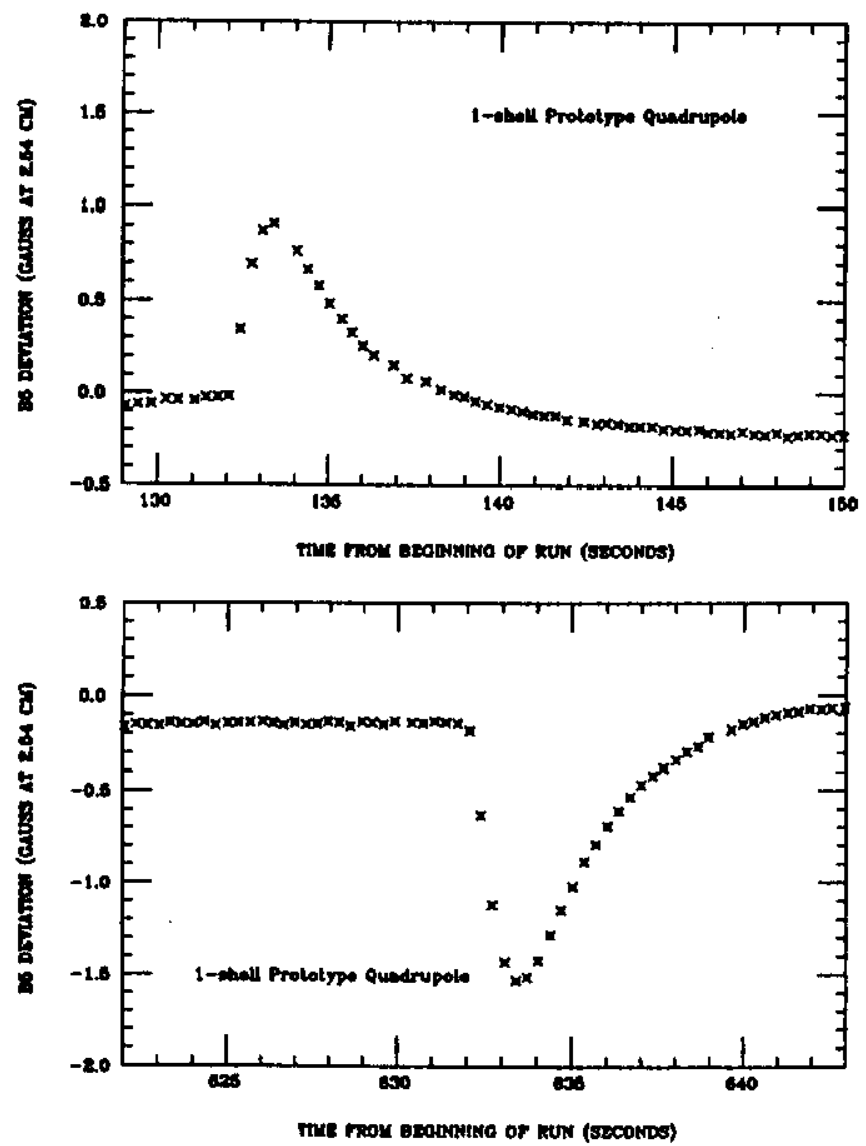

Figure 5. Difference of $\mathrm{B}_{5}$ ( due to ramp cycle with wait period of 500 seconds at 300 amperes ) and $B_{5}$ (due to uninterrupted ramp cycle ) as a function of time. Bs difference at the (a) begining (b) end of wait period.
3 For the remnant field studies, magnets were ramped in a sawtooth fashion three times to a maximum current of 1500 amps. Figure 6a shows the quadrupole $\left(\mathrm{B}_{1}\right), 12$-pole $\left(\mathrm{B}_{5}\right)$ and 20-pole (Bg) for the prototype magnet at $25 \mathrm{~A} / \mathrm{s}$. Each curve is fit to the sum of a constant, a $\log (t)$ and an exponential decay. The fit parameters are shown in Table 1. Each harmonic shows an exponential decay with approximately the same time constant. Figure $6 \mathrm{~b}$ shows the remnant $\mathrm{B}_{5}$ as a function of time at different ramp rates. The exponential time constants are independent of ramp rate, and the amplitude of the decay is proportional to the ramp rate. For the production magnet data, tests were performed at $6 \mathrm{~A} / \mathrm{s}$ only. No exponential decay was observed.
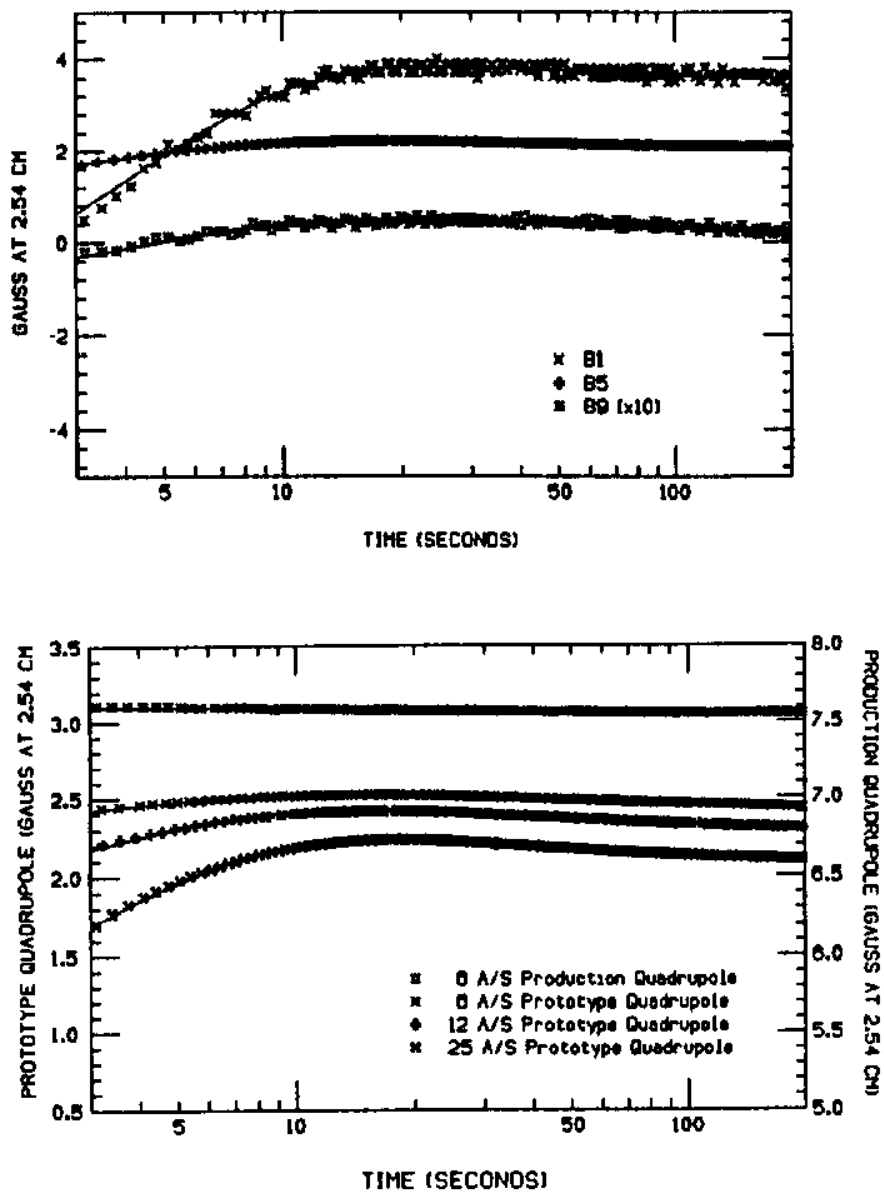

Figure 6. Remnant fields as a function of time, where $t=0$ is defined as the time when the transport field reaches zero current.a) Prototype 5-shell Quadrupole $\left(B_{1}\right), 12$ pole $\left(B_{5}\right)$ and 20 pole $\left(B_{9}\right)$ for $25 \mathrm{~A} / \mathrm{s}$ ramp cycle. b) Prototype (Bs) for $6 a / s, 12 a / s$ and $25 a / s$, and production magnet $\left(\mathrm{B}_{5}\right)$ for $6 \mathrm{~A} / \mathrm{s}$. Superimposed over each distribution is a fit of the form $A 0+A 1^{*} \exp (-t / A 2)+A 3^{*} \ln (t)$.

\section{TABLE 1}

Fit parameters for Remnant Fields as a function of time: $B_{n}=A_{0}+A_{1} * \exp \left(V A_{2}\right)+A_{3} * \ln (t)$

\section{1-Shell Prototype Ouadrupole:}

\begin{tabular}{|c|c|c|c|c|c|}
\hline $\begin{array}{l}\text { Harmonics } \\
\mathrm{B}_{1} \\
\mathrm{~B}_{9} \\
\mathrm{~B}_{4}\end{array}$ & $\begin{array}{l}\text { Ramo Rate } \\
25 \mathrm{~A} / \mathrm{s} \\
25 \mathrm{~A} / \mathrm{s} \\
25 \mathrm{~A} / \mathrm{s} \\
12 \mathrm{~A} / \mathrm{s} \\
6 \mathrm{~A} / \mathrm{s}\end{array}$ & $\begin{array}{c}\frac{\mathrm{A}}{4} \\
4.2+\% .11 \\
.08+\% .05 \\
2.4+\% .02 \\
2.6+-.02 \\
2.6+-.02\end{array}$ & $\begin{array}{c}\mathrm{Al} \\
-7.5+-.6 \\
-.22+-.1 \\
-1.7+-.1 \\
-0.9+-.1 \\
-0.4+-.1\end{array}$ & $\begin{array}{c}\frac{\mathrm{A}}{2} \\
4.1+1-.3 \\
4.0+-3.0 \\
3.3+-.02 \\
3.2+-.03 \\
3.5+1-.1\end{array}$ & $\begin{array}{c}\frac{\mathrm{A} 3}{} \\
-.12+/-.02 \\
-.01+/-.01 \\
. .06+/-.01 \\
-.05 /+.01 \\
-.04 /+.01\end{array}$ \\
\hline
\end{tabular}

1-Shell Production Ouadrupole:

$\frac{\text { Harmonics }}{\mathrm{B}_{5}} \frac{\text { Ramp Rate }}{6 \mathrm{~A} / \mathrm{s}} \underset{7.6+1-.01}{\stackrel{\mathrm{AO}}{2}}$


Time dependent magnetic fields have been observed in high gradient quadrupoles for the Tevatron Low Beta project. The 2-shell quadrupoles exhibit behavior reminiscent of Tevatron dipoles. For the measured allowed harmonics at constant currents, we observed changes in magnetic field that approximate $\log (t)$. The magnets rejoin the hysteresis curve shortly after the ramp cycle is continued. The 1shell magnets also show evidence for $\log (t)$ behavior. Additionally, we observed other time dependent behavior associated with changing the transport current from a constant ramp rate to constant current, and from constant current to constant ramp rate. Within 1 second of the ramp rate change, there is a sudden change in B5, followed by an exponential decay. This effect is more pronounced in the 1-shell prototype which has a larger $\mathrm{Cu} / \mathrm{Sc}$ ratio and larger filament spacing/diameter. The level of the change is proportional to ramp rate and the subsequent decay has an approximately a 3 second decay constant for the prototype. This time constant is are comparable for all ramp rates and for all measured allowed harmonics. This suggests that these exponential field decays are generated by Eddy currents which are coupled to the transport current magnetic field. However the existence of Eddy currents does not adequately explain the sign of the remnant field quadrupole decay which increases in sign after the transport current is stopped. It also does not explain the observed sudden change in $B_{5}$ before the 3 second exponential decay. Further studies on these magnet designs are needed to fully understand these phenomena.

\section{Acknowledgement}

We would like to thank the Fermilab Technical Support Section, in particular the Superconducting Magnet R \&D facility, for their assistance in making this test possible. We would also like to thank Moyses Kuchnir and Alvin Tollestrup for their discussions on Eddy currents in superconducting magnets.

\section{REFERENCES.}

1. A. D. McInturff et al., "The Fermilab collider D0/Low Beta System", European Particle Accelerator Conference, Rome, Italy, p. 1264, 1988

2. R. W. Hanft et al., "Studies of Time Dependence of Fields in Tevatron Superconducting Dipole Magnets", March 1989. IEEE Transactions on Magnetics, Vol. 25. No. 2, March 1989, P. 1647

3.

D. A. Herrup et al., "Time Variations of Fields in Superconducting Magnets and Their Effects on Accelerators", IEEE Transactions on Magnetics, Vol. 25. No. 2, March 1989 P. 1643

4. P. M. Mantsch et al.,"A New High-Gradient Correction Quadrupole for The Fermilab Luminosity Upgrade", IEEE Particle and Accelerator Conf., Chicago, II, March 20-23 1989, p 512.

5. M. J. Lamm et al, "Test of High Gradient Superconducting Quadrupole Magnets for the Tevatron", Advances in Cryogenic Engineering, Vol. 35A. P609., 1989

6. "Tevatron Low-Beta Quadrupole Specification", February 15,1990

7. K. McGuire, et al., "Cryogenic Instrumentation of an SSC Magnet Test Stand", Adv. Cryo. Engr. 33, 1063 (1988)

8. J. Strait, et al., "Fermilab R\&D Test Facility for SSC Magnets",Presented at the 1989 International Industrial Symposilum on the Super Collider, New Orleans, LA, February 8-10, 1989 (Fermilab preprint TM-1563)

9. G. H. Morgan. "Stationary coil for Measuring the Harmonics in Pulsed Transport Magnets", Proc. Inter.Conf. on Magnet Technol, edited by Y. Winterbottom p.787, 1972 